Cipango Cahiers d'études japonaises

Hors-série | 2008

Autour du Genji monogatari

\title{
Le clos du Paulawnia (traduction)
}

Le Roman du Genji

The Paulownia Court: translation

\section{Murasaki Shikibu}

\section{CpenEdition}

Journals

Édition électronique

URL : https://journals.openedition.org/cipango/586

DOI : $10.4000 /$ cipango.586

ISSN : 2260-7706

Éditeur

INALCO

Édition imprimée

Date de publication : 1 janvier 2008

Pagination : 13-37

ISBN : 978-2-85837-170-5

ISSN : $1164-5857$

Référence électronique

Murasaki Shikibu, «Le clos du Paulawnia (traduction) », Cipango [En ligne], Hors-série | 2008, mis en ligne le 01 mai 2012, consulté le 30 juin 2021. URL : http://journals.openedition.org/cipango/586 ; DOI : https://doi.org/10.4000/cipango.586

Ce document a été généré automatiquement le 30 juin 2021.

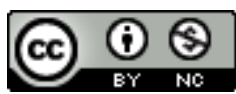

Cipango est mis à disposition selon les termes de la Licence Creative Commons Attribution - Pas d'Utilisation Commerciale 4.0 International. 


\title{
Le clos du Paulawnia (traduction)
}

\author{
Le Roman du Genji \\ The Paulownia Court: translation
}

\section{Murasaki Shikibu}

1 En quel règne était-ce donc? - parmi les nombreuses épouses impériales et dames d'atour au service de Sa Majesté, il en était une qui, sans être de naissance insigne, jouissait plus que toute autre de la faveur du souverain. Les épouses, persuadées chacune depuis toujours que la préférence lui était due, en étaient outrées : aussi l'accablaient-elles de leur dédain et de leur jalousie. Les dames d'atour de même rang ou de condition inférieure se sentaient, quant à elles, plus menacées encore. La voir ainsi du matin au soir en service auprès de l'empereur exacerbait le ressentiment général ; était-ce le poids de toute cette haine accumulée, elle se mit à dépérir et, dans sa détresse, à se retirer de plus en plus souvent dans sa demeure familiale. Mais loin de se détourner d'elle, le souverain ne l'en aimait que davantage et restait sourd aux critiques, au risque de donner par son comportement un exemple déplorable. Hauts dignitaires et courtisans eux-mêmes ne pouvaient s'empêcher de détourner des regards offusqués. La faveur qu'il lui témoignait était scandaleuse. En Chine, c'était pour des affaires de cette sorte que le monde avait connu des troubles dont il n'avait rien résulté de bon. Le mécontentement et l'inquiétude grandissaient dans l'empire, et l'on n'était plus loin de citer le précédent de Yang Guifei ${ }^{1}$. Pourtant, bien qu'en butte à de nombreuses vexations, la dame continuait à séjourner au palais avec pour seul appui l'affection sans pareille que daignait lui prodiguer sa Majesté.

2 Le grand conseiller son père était décédé ; sa mère, l'épouse principale, en héritière du raffinement des gens d'autrefois, l'avait pourvue de tout le nécessaire de façon à ce que, lors des diverses célébrations à la cour, elle ne le cédât en rien aux dames dont la renommée était pour l'heure la plus éclatante et qui bénéficiaient du soutien de leurs deux parents. Cependant, comme aucun protecteur digne de ce nom n'était là pour veiller sur elle, elle semblait, dans les grandes occasions, d'autant plus vulnérable qu'elle se trouvait sans appui. 
3 Sans doute, les liens noués par l'empereur et sa favorite dans une vie antérieure étaient-ils profonds, car un prince leur naquit, à la splendeur sans pareille. Brûlant d'impatience, le souverain le fit au plus tôt présenter au palais: l'enfant était d'une exceptionnelle beauté. Le prince premier, né d'une épouse impériale, fille du ministre de droite, bénéficiait d'appuis solides, et comme il ne faisait aucun doute qu'il deviendrait prince héritier, il était l'objet de tous les soins, de toutes les attentions. Mais comment aurait-il pu rivaliser avec l'éclat de son frère? Si l'empereur traitait l'aîné avec tous les égards dus à son rang, il voua au prince cadet une passion exclusive, l'entourant d'une sollicitude infinie.

4 Par sa naissance, la favorite était d'une condition trop élevée pour le service ordinaire du palais. Jouissant dans le monde de la plus grande considération, elle avait tout d'une personne de haute qualité. Mais le souverain exigeait sa présence à ses côtés plus que de raison: c'est elle qu'il faisait venir à chaque divertissement musical de quelque importance ou à l'occasion de tout événement marquant. Parfois un réveil tardif lui donnait l'occasion de la garder à son service sans interruption. Ainsi l'avait-il dépréciée aux yeux du monde en lui imposant de demeurer sans cesse auprès de lui. Or depuis la naissance du prince, il avait redoublé d'égards envers elle si bien que l'épouse principale, mère du prince premier, conçut des soupçons: l'empereur n'irait-il pas jusqu'à faire de cet autre enfant le prince héritier? Le souverain lui témoignait l'estime particulière qu'il devait à celle qui était entrée la première au palais, et comme elle lui avait en outre donné plusieurs enfants, ses reproches ne laissaient pas de susciter en lui contrariété et embarras.

5 La favorite pouvait, certes, compter sur la protection de Sa Majesté, mais nombreux étaient ceux qui guettaient ses moindres défaillances afin de l'humilier, et pour elle, si fragile, la faveur impériale elle-même était devenue source de tourment. Elle avait pour logis au palais le Kiritsubo². Comment s'étonner que les allées et venues incessantes du souverain, qui passait sans daigner s'y arrêter devant leurs appartements, aient exaspéré ses nombreuses épouses? Aussi, lorsque les visites de la favorite à l'empereur se faisaient vraiment trop fréquentes, lui jouait-on ici et là de fort vilains tours, sur les passerelles ou les galeries, et il arrivait même que le bas des robes de ses dames de compagnie portât les traces d'ignobles outrages. Par ailleurs, il n'était pas rare qu'on s'arrangeât pour l'humilier en l'enfermant dans le couloir qu'elle ne pouvait éviter d'emprunter et dont on verrouillait les portes devant et derrière elle. Comme à tout propos les tourments qu'on lui infligeait ne faisaient que se multiplier, la plongeant dans une affliction sans bornes, l'empereur en ressentit une profonde pitié: il fit transférer ailleurs les appartements de la dame d'atour qui résidait au Kōrōden ${ }^{3}$ pour les attribuer à sa favorite, nourrissant plus encore la haine implacable dont elle était l'objet.

6 L'année où le prince eut trois ans, pour la cérémonie de la prise de l'habit de cour, l'empereur lui fit tenir de somptueux vêtements, qui ne le cédaient en rien à ceux qu'avait portés le prince premier à la même occasion, puisant pour ce faire dans les plus beaux trésors de l'office des magasins de la cour et de la chancellerie privée. Les critiques allèrent bon train ; pourtant la beauté et les qualités de l'enfant, sa précocité, apparaissaient si rares, si extraordinaires qu'on ne pouvait se résoudre à le détester. Les gens d'expérience écarquillaient les yeux, stupéfaits qu'il pût exister en ce monde un être à ce point parfait. 
7 L'été de cette même année, la mère du prince, qui se sentait souffrante, voulut se retirer dans sa famille, mais l'empereur ne l'autorisa pas à s'absenter. Comme sa santé avait toujours été fragile, il s'était habitué à la voir ainsi et se contenta de lui dire : "Attendons encore un peu!", mais son mal ne cessa de s'aggraver au point qu'en cinq ou six jours, elle était devenue extrêmement faible. Sa mère, éplorée, s'adressa alors au souverain et obtint de la ramener chez elle. Dans la crainte que, même en pareille circonstance, quelque nouveau scandale n'éclatât, la favorite s'apprêta à se retirer secrètement en laissant le prince au palais. Contraint de se plier aux usages, l'empereur dut finalement la laisser partir et l'impossibilité où il était ne serait-ce que d'assister à son départ le plongea dans une indicible tristesse. Elle, naguère si resplendissante, si pleine de charme, avait maintenant le visage émacié, et à la voir ainsi sur le point de s'éteindre, encore en vie ou déjà morte, trop faible pour exprimer par la parole l'émotion qui l'envahissait, le souverain, oubliant passé et avenir, lui faisait en sanglotant serment sur serment auxquels elle n'avait plus même la force de répondre, et comme elle gisait là, si frêle, le regard vacillant, à peine consciente, éperdu il ne savait plus que faire. Après avoir donné l'ordre de préparer le palanquin ${ }^{4}$, il revint encore une fois auprès d'elle et, ne pouvant toujours pas se résoudre à la laisser partir : «Vous m'aviez promis, dit-il, de ne pas me précéder sur le chemin de l'ultime voyage ! Comment pouvez-vous m'abandonner ainsi? ». Elle lui jeta alors un regard empreint d'un désespoir infini, et

Grande est la douleur

d'avoir à se séparer

au terme de ce chemin :

combien j'eusse aimé continuer

à cheminer auprès de Vous!

8 Si j'avais pensé qu'il en serait ainsi, je... ", dit-elle : le souffle de plus en plus faible, elle semblait vouloir poursuivre, sans pourtant trouver la force de surmonter sa souffrance et son épuisement. L'empereur se promit alors de rester à ses côtés jusqu'à la fin, mais on vint l'avertir à ce moment que les moines appelés afin de prononcer les déprécations prévues pour commencer ce même jour officieraient dès le soir, et comme on insistait, il dut la laisser partir le cœur brisé. La poitrine oppressée, incapable de fermer les yeux un seul instant, il restait là à attendre l'aube. Dans son impatience à voir revenir le messager qu'il avait dépêché, il ne cessait de dire son inquiétude, quand celui-ci se présenta, le visage défait: "Au moment où l'on passait la mi-nuit, elle a cessé de vivre ", avait-il appris là-bas au milieu des pleurs et des cris. Rendu fou de douleur par cette nouvelle, le souverain, l'esprit égaré, s'enferma dans ses appartements.

9 Malgré les circonstances, l'empereur aurait souhaité garder le jeune prince auprès de lui, mais comme il n'y avait aucun précédent à cela, on commença à préparer son départ. L'enfant ne comprenait rien à ce qui se passait autour de lui : le chagrin qui accablait les dames en service au palais, les larmes que versait sans fin Sa Majesté, tout lui semblait étrange. Comment pareille séparation pourrait-elle ne pas être douloureuse? Mais dans le cas présent, elle était poignante au-delà de toute expression.

10 Le temps venu, il fallut bien se résoudre à procéder aux cérémonies d'usage. La mère de la défunte, se consumant de chagrin au point de souhaiter disparaître avec elle en une même fumée, voulut à tout prix monter dans l'une des voitures occupées par les dames du cortège funèbre, mais lorsque l'on arriva au lieu dit Otagi où se déroulait cette célébration des plus solennelles, de quels sentiments son cœur pouvait-il bien être 
agité ? «À voir son corps sans vie, j'ai le sentiment qu'elle est encore de ce monde, mais à quoi bon? C'est quand je la verrai réduite en cendres que je pourrai enfin me convaincre qu'elle est morte ", avait-elle dit avec fermeté, mais à présent qu'elle était là, défaite, tout près de tomber de la voiture, les autres dames, qui se doutaient bien de la tournure que prendraient les choses, s'en trouvèrent fort embarrassées. Un messager arriva alors du palais. Il était porteur d'un ordre de l'empereur qui élevait la défunte au troisième rang, et la lecture solennelle de ce décret ajouta encore à la tristesse. Le souverain ne lui avait pas même de son vivant conféré le titre d'épouse impériale, et il en éprouvait d'infinis regrets ; aussi, avait-il tenu à la promouvoir ne fût-ce que d'un rang. À cette occasion encore, nombreuses furent celles qui en conçurent du ressentiment. Cependant, celles de ses rivales qui avaient du discernement reconnaissaient à présent combien son allure et ses traits étaient gracieux, et combien son caractère doux et plaisant la rendait impossible à haïr. On l'avait jalousée sans pitié à cause des faveurs déplacées de l'empereur, mais les dames au service de Sa Majesté pensaient aujourd'hui avec regret à son charme et à la bonté de son cœur. Ne dit-on pas : «C'est une fois disparue que...»?

11 Les jours passaient, et le souverain ne manquait pas de s'associer aux offices funèbres. À mesure que le temps s'écoulait, un chagrin irrémédiable l'étreignait, si bien que, ne faisant plus venir ses épouses auprès de lui, il passait jours et nuits à répandre des pleurs et pour tous ceux qui le voyaient ainsi, l'automne fut également mouillé de larmes. "Quel attachement est-ce là, dont même la mort ne peut nous délivrer !", s'indignait la dame du Kokiden ${ }^{5}$, toujours en proie au ressentiment. La vue du prince premier ne faisait qu'aviver la douleur de l'empereur à qui son plus jeune fils manquait cruellement, et souvent il envoyait une dame de son entourage ou sa nourrice s'enquérir de lui.

12 Après les premières bourrasques d'automne, un soir que le temps avait soudain fraîchi, le souverain, assailli de souvenirs plus encore que de coutume, dépêcha chez la mère de la défunte la dame répondant au nom de Yugei ${ }^{6}$. La lune resplendissait dans le ciel quand il la fit partir et lui demeura là, perdu dans ses pensées. Autrefois, lorsqu'en de tels moments ils s'adonnaient à la musique, elle tirait des sons merveilleux des cordes de son instrument, les plus anodines de ses paroles étaient d'une beauté singulière, et s'il croyait ainsi deviner à ses côtés sa silhouette à nulle autre pareille, cette vision n'avait pas même la réalité d'« une rencontre fugitive au sein de la nuit ${ }^{7}$ ».

Arrivée là-bas, Yugei découvrit, une fois passé le portail, un spectacle désolant. Malgré son veuvage, la dame mère s'était efforcée par égard pour son unique fille d'entretenir sa demeure et était parvenue à la maintenir dans un état décent, mais maintenant qu'elle restait là, plongée dans les ténèbres du désespoir, les herbes avaient poussé haut dans le jardin, et après les bourrasques d'automne, l'endroit paraissait encore plus sauvage, éclairé seulement par les rayons de la lune que n'arrêtait pas l'enchevêtrement des broussailles. La visiteuse fut introduite par l'entrée principale de la demeure ${ }^{8}$. La dame mère, tout aussi émue, fut d'abord incapable de prononcer un seul mot. Prenant enfin la parole : « Lui survivre si longtemps m'est déjà insupportable, dit-elle, que dire de la confusion où me plonge la venue d'une messagère de votre qualité, daignant se frayer un chemin parmi ces armoises couvertes de rosée?», puis cédant à l'émotion, elle se mit à pleurer. "La maîtresse en second du palais avait confié à Sa Majesté que, lors de sa venue ici-même, elle avait senti son cœur se briser à la vue de votre détresse : aussi fruste que je sois, je ne puis à mon tour rester insensible à 
votre affliction ", lui répondit Yugei qui, s'étant quelque peu ressaisie, entreprit de transmettre le message impérial : « “N'est-ce qu'un rêve, me suis-je demandé un temps, éperdu, mais tandis que peu à peu mon trouble s'apaise, je n'ai pas même à qui m'ouvrir de ce tourment insupportable dont nul réveil ne peut me délivrer: si seulement sa mère pouvait me rendre secrètement visite... Je m'inquiète aussi pour le jeune prince, le savoir passer ses journées au milieu des larmes de rosée me plonge dans l'affliction : qu'elle vienne donc avec lui au plus vite." Devant le douloureux spectacle de Sa Majesté, prise entre les sanglots qui l'étouffaient et la crainte de paraître faible aux yeux de son entourage, j'ai dû me retirer sans qu'elle ait pu m'en donner à entendre davantage ", dit Yugei en remettant la lettre de l'empereur. "Tout aveuglée que je sois par mes larmes, les augustes paroles de Sa Majesté m'éclaireront ", répondit la dame mère, puis elle se mit à lire. «Avec le temps, le chagrin ne s'atténuerait-il pas quelque peu ? C'est avec cet espoir que je vois passer les jours et les mois, or inexplicablement la douleur demeure, insupportable. Comment va l'enfant, me demandé-je sans cesse, et ne pouvoir être avec vous pour l'élever me soucie: acceptez de venir avec lui, en souvenir du passé ", avait écrit le souverain, dans son infinie sollicitude.

Lande de Miyagi,

la rosée perle

au vent qui bruit

et pousse mes pensées

vers le jeune lespédèze

ajoutait-il, mais elle fut incapable de poursuivre la lecture jusqu'à la fin. «Moi qui apprends à mes dépens combien est douloureuse une si longue vie, comment oserais-je monter au palais quand la seule idée de ce que pourrait penser «le pin de Takasago ${ }^{9}$ me remplit de confusion? Je ne saurais m'y résoudre, bien que Sa Majesté ait daigné m'y mander à plusieurs reprises. Que comprend le jeune prince, je ne sais, mais il semble avoir hâte de se rendre au palais, et comment pourrait-il en être autrement?, me dis-je en le contemplant pleine de tristesse : telles sont les pensées qui m'occupent et que je vous saurais gré de lui transmettre. Que le prince vive ainsi, en ma funeste compagnie, est pour lui un sinistre présage, et pour moi un honneur dont je suis indigne ", dit-elle.

L'enfant était déjà endormi. "J'aurais souhaité voir Son Altesse pour rapporter des nouvelles détaillées, mais Sa Majesté m'attend, et la nuit est déjà bien avancée », dit Yugei en s'apprêtant à partir. "J'aimerais m'entretenir encore avec vous afin de dissiper, ne serait-ce qu'un peu, les ténèbres insupportables dans lesquelles mon cœur s'égare, lui répondit la dame mère. Revenez me voir, je vous en prie, à titre personnel et nous parlerons à loisir. Des années durant, vous m'avez rendu visite en des occasions heureuses et fastes : vous recevoir aujourd'hui porteuse d'un pareil message, c'est bien, hélas, l'effet d'un cruel destin! Notre fille était depuis sa naissance l'objet de tous nos espoirs, et feu le grand conseiller m'avait maintes et maintes fois exhortée, et ce, jusque dans ses ultimes paroles: "Mon vœu le plus cher est qu'elle serve au palais. Faites en sorte qu'il se réalise sans faute. Même après ma disparition, n'y renoncez pas.” Je n'ignorais pas qu'il serait périlleux pour elle de se trouver à la cour sans protecteur influent, et pourtant, je l'y fis entrer, soucieuse de ne pas contrarier les dernières volontés de mon époux. Tandis que Sa Majesté l'honorait de mille faveurs insignes, ma fille s'acquittait de son service, dissimulant la honte que faisait naître en elle le mépris dont elle était l'objet, mais le ressentiment à son égard alla croissant, les avanies se multiplièrent au point d'entraîner sa disparition prématurée, et c'est pourquoi, ces 
augustes faveurs, j'en suis venue à les considérer comme bien cruelles. Mais, sans doute, est-ce là l'effet des ténèbres qui plongent mon cœur dans la confusion. » Elle s'interrompit, la voix brisée par les sanglots. Il se faisait tard. «Sa Majesté éprouve les mêmes sentiments: "Si, cédant à l'inclination de mon cœur, je l'ai aimée d'une telle passion qu'on a pu s'en émouvoir, c'est que le lien qui nous unissait était condamné à se rompre, et aujourd'hui, il ne m'apporte que douleur. Je crois n'avoir jamais en quoi que ce soit blessé personne, et pourtant, pour l'avoir aimée, je me suis attiré le ressentiment de toutes celles dont le rang exigeait qu'on les ménageât, si bien que, finalement, je me retrouve abandonné, sans pouvoir trouver d'apaisement, et réduit plus que jamais à faire figure d'insensé, objet de la réprobation générale. Qu'a-t-il donc pu se passer dans nos existences antérieures?", ne cesse-t-il de se demander, en trempant ses manches de ses larmes. " Yugei avait encore beaucoup à dire. «La nuit est très avancée » dit-elle sans cesser de pleurer, en se levant pour partir, « et il me faut transmettre votre réponse avant qu'il ne soit trop tard. » La lune allait se coucher dans un ciel limpide, le vent avait beaucoup fraîchi, le chant des insectes dans les buissons appelait les pleurs, et il lui était difficile de s'arracher à cette demeure envahie par les herbes.
Jusqu'à l'épuisement
crie le grillon
et comme lui,
en cette longue nuit,
sans fin, je verse des larmes

La visiteuse ne pouvait se résoudre à monter dans sa voiture. Et la dame mère de lui faire répondre :

"Assourdissant résonne

le chant des insectes

dans les herbes folles

où de ses larmes dépose la rosée

la dame du céleste séjour

irai-je jusqu'à vous en faire reproche? » Les circonstances ne se prêtant pas à l'offre de présents de choix, elle avait simplement réuni des souvenirs de la disparue : une tenue de cour au complet, soigneusement conservée pour une telle occasion, à laquelle elle avait joint quelques objets, dont un nécessaire de coiffure. Les jeunes dames de compagnie de la défunte éprouvaient bien sûr une peine immense. Elles s'étaient en outre accoutumées à la vie du palais et en nourrissaient une vive nostalgie, aussi à chaque fois qu'était évoqué le souvenir de Sa Majesté, ne manquaient-elles pas de suggérer que le prince reparût au plus tôt à la cour. Mais la dame mère se disait que la présence auprès de l'enfant au palais d'une personne marquée par le malheur comme elle donnerait lieu à des médisances; en même temps, l'idée de ne pouvoir veiller sur lui, même un bref instant, la plongeait dans une telle inquiétude qu'elle ne pouvait se résoudre à le laisser partir.

De retour au palais, Yugei eut le cœur serré en trouvant l'empereur encore éveillé. Sous le prétexte d'admirer le jardin clos, alors au plus fort de sa beauté, il avait fait venir auprès de lui quatre ou cinq dames choisies pour leur exquise sensibilité, avec lesquelles il s'entretenait à mi-voix. À cette époque, il contemplait du matin au soir le paravent du Chant des regrets éternels ${ }^{10}$ : les peintures, les poèmes que l'empereur retiré Teiji-no-in ${ }^{11}$ avait fait composer par Ise et Tsurayuki et qu'il avait calligraphiés de sa propre main ${ }^{12}$, ainsi que le poème chinois lui-même ne cessaient de revenir dans sa conversation. Il pressa de questions Yugei qui lui fit part en confidence de la situation 
poignante qu'elle avait découverte. L'empereur parcourut la réponse qui lui était adressée : "La lettre de Sa Majesté me remplit de confusion et la volonté qu'elle exprime plonge mon cœur dans les ténèbres du désarroi.

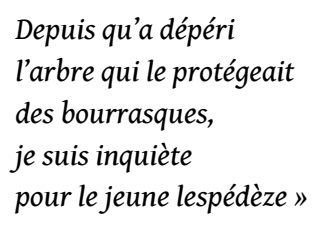

Propos dont le souverain ne pouvait sans doute que pardonner l'irrévérence, y voyant la marque d'un cœur égaré par le chagrin. Il chercha en vain à maîtriser le trouble qui l'envahissait, soucieux de ne pas le laisser voir, mais ne put cacher sa douleur. Comme il rassemblait les souvenirs des années qu'il avait passées avec sa bien-aimée, depuis les tout premiers temps de leur rencontre, et qu'il en revoyait chaque instant, il frémit à l'idée que tant de jours et de mois s'étaient maintenant écoulés sans elle, alors que de son vivant la moindre de ses absences lui était insupportable. "Afin de la remercier de la persévérance dont elle a fait preuve pour accomplir la volonté du grand conseiller et envoyer sa fille au palais, j'ai toujours voulu faire en sorte qu'elle pût se féliciter de cette décision. Mais à quoi bon en parler désormais ?... », dit l'empereur en pensant avec émotion à la dame mère. "Et pourtant, ajouta-t-il, de telles occasions se présenteront sans doute d'elles-mêmes une fois que le jeune prince aura grandi. Qu'elle ait pour seul souci de vivre encore longtemps ». Yugei montra à Sa Majesté le présent envoyé par la dame mère. "Que n'est-ce une épingle à cheveux, preuve qu'elle aurait trouvé la demeure de celle qui n'est plus ${ }^{13}$ !», songea l'empereur, toujours en vain.

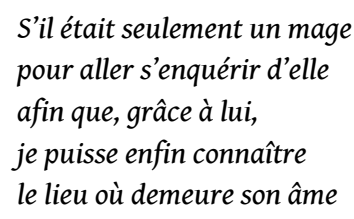

Le portrait peint de Yang Guifei était dû à un artiste hors pair, mais l'art du pinceau a ses limites et il lui manquait l'éclat de la vie. Elle avait dû être splendide dans sa parure à la mode chinoise, avec son visage semblable « aux lotus de l'étang de Taiyi » et ses sourcils pareils " aux saules du parc de Weiyang ${ }^{14}$ ", mais quand l'empereur se rappelait le charme empreint de douceur et de fragilité de la défunte, il ne trouvait ni fleurs ni oiseaux auxquels il pût la comparer ${ }^{15}$. Ils s'étaient tant de fois promis, au cours de leurs tendres échanges, d'être "deux oiseaux n'ayant qu'une seule paire d'ailes", "deux arbres partageant une même ramure ${ }^{16}$ " mais sa vie trop brève avait rendu vains ces serments et il en éprouvait un chagrin infini ${ }^{17}$. Tandis qu'il restait plongé dans les tristes pensées qu'éveillaient en lui le bruit du vent ou le chant des insectes, la dame du Kokiden, qui ne se rendait plus depuis longtemps dans ses appartements du Seiryōden ${ }^{18}$, prenait prétexte de la beauté de la lune pour se livrer, jusque tard dans la nuit, à quelque divertissement musical dont on percevait les échos. Le souverain en éprouvait un déplaisir extrême. Témoins de ses souffrances, les courtisans, hommes ou femmes, écoutaient eux aussi consternés. Cette princesse, qui se distinguait par un caractère intransigeant et autoritaire, se comportait comme si rien ne s'était passé. La lune avait maintenant disparu.

Même au-dessus des nuages / Au sein même du palais les yeux sont noyés de larmes / voici qu'elle se couche la lune d'automne; 
comment luirait-elle claire / comment peut-on vivre

dans la demeure envahie par les herbes ? ${ }^{19}$ «des lampes dont les mèches achevaient de se consumer ${ }^{20}$ ». On entendait la voix des officiers de la garde de droite venus prendre leur quart: ce devait être l'heure du bœuf $^{21}$. Le souverain, soucieux des regards, finissait par se retirer dans sa chambre, mais « ne trouvait toujours pas le sommeil ». Il avait beau se lever dès l'aurore, lorsqu'il se souvenait de ces temps où "ils ignoraient l'aube ${ }^{22}$ ", sans doute se sentait-il toujours aussi peu enclin à tenir audience. Il ne prenait guère non plus de nourriture, touchait à peine à la collation du matin, ne montrait pas plus d'appétit lors du Grand Repas, et les courtisans préposés à la Table se désespéraient de le voir dans un état aussi misérable. Tous ceux, hommes et femmes, qui étaient à son service se lamentaient de concert, avouant leur désarroi devant cette situation des plus embarrassantes. Était-ce à la suite de quelque lien contracté dans une existence antérieure ?, il avait tenu pour rien les critiques et le ressentiment du grand nombre, s'écartant de la raison pour tout ce qui touchait à la favorite, et maintenant qu'elle avait disparu, voilà qu'il perdait tout intérêt pour les affaires de ce monde! Ainsi, se lamentait-on, scandalisé, n'hésitant pas à citer à voix basse les précédents des cours étrangères.

21 Jours et mois passant, le jeune prince était entré au palais. Sa précocité et sa beauté n'étaient pas d'un être de ce monde, ce qui inspirait au souverain les plus vives inquiétudes sur son sort. Au printemps de l'année suivante, l'empereur souhaita ardemment l'avantager au détriment de son frère aîné à l'occasion de la désignation du prince héritier. Cependant, outre que l'enfant ne disposait d'aucun appui à la cour, c'était là un dessein fort peu susceptible de recueillir l'assentiment des gens, et Sa Majesté, craignant que cette décision ne devînt au contraire dangereuse pour lui, avait décidé de n'en rien laisser paraître. "Quelle que soit la faveur qu'il lui témoigne, il y a une limite à tout!», en conclut-on dans le monde, et l'épouse impériale de se rasséréner. Quant à la dame mère, toujours plongée dans un désespoir sans remède, son vœu de rejoindre la défunte là où elle devait désormais se trouver aurait-il finalement été exaucé ? Elle mourut, et le souverain éprouva à nouveau une profonde tristesse. Le jeune prince, qui allait vers ses six ans, comprit cette fois ce qui se passait et pleura cette disparition. De son côté, elle avait exprimé maintes et maintes fois son chagrin de laisser derrière elle l'enfant que pendant tant d'années elle avait entouré de son affection.

Désormais le prince résidait au palais. Quand il eut sept ans, l'empereur fit célébrer en son honneur la cérémonie de la Première lecture ${ }^{23}$. Devant la sagesse et le discernement sans pareils de l'enfant, il ne pouvait s'empêcher d'éprouver de l'effroi. Songeant que personne ne saurait le haïr désormais, «Veuillez le chérir maintenant que le voilà sans mère ", disait-il lorsqu'il l'emmenait avec lui dans ses visites y compris au Kokiden où l'enfant était volontiers accueilli derrière les stores. Sa beauté était telle que sa seule vue aurait arraché un sourire au guerrier le plus féroce, à l'ennemi le plus acharné, aussi l'épouse impériale était-elle incapable de le rejeter. Les deux jeunes princesses, ses propres filles, ne pouvaient lui être comparées. Aucune dame ne se serait cachée de lui. Impressionnées par le charme qui se dégageait de sa personne, toutes voyaient en lui un compagnon plein d'attrait, mais quelque peu intimidant. Excellant - cela va sans dire - dans l'indispensable voie des Lettres, il ravissait le séjour des nuages ${ }^{24}$ par la 
musique de son luth ou de sa flûte, mais détailler ici tous ses talents risquerait d'être fastidieux.

À cette époque, le souverain entendit dire que parmi les envoyés de Koma ${ }^{25}$ présents à la cour se trouvait un physiognomoniste remarquable, mais comme, à cause de l'interdit formulé par l'empereur $\mathrm{Uda}^{26}$, il ne pouvait le faire venir au palais, il envoya le jeune prince au Kōrokan ${ }^{27}$ dans le plus grand secret. Quand le grand contrôleur de droite chargé d'escorter l'enfant le présenta en le faisant passer pour son fils, le physiognomoniste fut stupéfait et resta un long moment à hocher la tête, perplexe. Puis, il dit: "Voilà une personne qui présente tous les traits d'un futur père pour le pays, de quelqu'un qui doit parvenir au rang suprême d'empereur... Mais s'il devait en être ainsi, désordres et malheurs seraient à prévoir. À moins que l'on ne voie en lui un futur pilier de la cour et un soutien pour l'empire, mais dans ce cas, les signes ne concordent pas ". Le grand contrôleur étant, lui aussi un lettré au savoir exceptionnel, leur entretien fut de la plus haute tenue. Ils échangèrent des poèmes en chinois, et le physiognomoniste composa de manière brillante une pièce dans laquelle il décrivait sa joie d'avoir rencontré à la veille de son départ une personne aussi exceptionnelle que cet enfant, joie que la séparation ne manquerait pas de muer en tristesse. Le prince à son tour produisit des vers extrêmement émouvants que le physiognomoniste porta aux nues avant de lui faire des cadeaux somptueux. De nombreux présents parvinrent également de la cour. Cependant, l'affaire s'ébruita d'elle-même. Le souverain n'en avait soufflé mot, mais certains comme le ministre, grand-père du prince héritier, se demandèrent ce que cela signifiait et en conçurent des soupçons. L'empereur n'avait rien appris qu'il ne sût déjà, car dans sa prévoyance, il avait autrefois ordonné l'examen $\mathrm{du}$ prince par un physiognomoniste japonais, et c'est pourquoi il s'était abstenu à ce jour de lui décerner le titre de prince impérial ${ }^{28}$. Frappé par la perspicacité du physiognomoniste de Corée, il se dit qu'il ne devait pas mettre l'enfant dans la situation précaire d'un prince impérial sans rang, dépourvu de tout appui du côté de sa famille maternelle, et jugea, devant l'incertitude pesant sur la durée de son propre règne, que l'avenir serait mieux assuré s'il faisait de lui un soutien de la cour en qualité de simple sujet. Une fois cette décision prise, il le fit instruire plus soigneusement encore dans les différentes voies d'études ${ }^{29}$. Certes, il était regrettable de réduire à la condition de sujet un enfant doué d'aptitudes aussi exceptionnelles, mais, d'un autre côté, il était à craindre que s'il devenait prince impérial, il serait toujours l'objet de la méfiance générale ; un nouvel examen par un astrologue de grand talent ayant abouti aux mêmes conclusions, l'empereur résolut de lui conférer le nom de Genji.

Les mois et les années avaient beau passer, pas un instant le souverain ne cessait de penser à la défunte. Espérant adoucir sa peine, il faisait venir auprès de lui celles qui pouvaient prétendre à l'en distraire, mais pourquoi ne s'en trouvait-il point en ce monde qui souffrît la comparaison avec la disparue?, se disait-il, plongé dans le désespoir. Or, il y avait une princesse, quatrième fille d'un empereur précédent, hautement réputée pour sa grande beauté et élevée avec un soin extrême par sa mère l'impératrice. Une maîtresse en second au service du palais apercevait parfois la jeune princesse qu'elle connaissait depuis l'enfance, car, occupant déjà des fonctions au temps de cet empereur, elle avait ses entrées chez l'ancienne impératrice. «J'ai servi au palais pendant trois règnes successifs sans avoir jamais rencontré personne qui égalât en beauté la défunte dame, dit-elle à Sa Majesté, mais cette princesse lui ressemble chaque jour davantage à mesure qu'elle grandit. C'est un être d'une rare beauté ». Ces paroles éveillèrent la curiosité du souverain qui invita instamment la princesse à venir 
au palais. La mère de celle-ci en fut épouvantée. Elle se rappelait avec effroi l'extrême méchanceté de l'épouse impériale mère du prince héritier, le mépris dont avait été publiquement l'objet la dame d'atour du clos du Paulownia et, toute à son inquiétude, elle ne pouvait se résoudre à obtempérer. Les choses en étaient là lorsqu'elle mourut. L'empereur réitéra alors son invitation d'une manière encore plus pressante à celle que la perte de sa mère avait plongée dans la plus grand détresse. "Laissez-moi vous considérer à l'égal des princesses mes filles ${ }^{30} »$. Les dames à son service, les gens de sa parentèle, son frère aîné, le prince chef du département des affaires militaires, la firent entrer au palais, estimant qu'elle trouverait plus de consolation en vivant à la cour que dans la situation précaire où elle était. On l'appela désormais Fujitsubo, la « dame du clos de la Glycine ». À la vérité, sa beauté et sa manière d'être rappelaient à l'empereur la défunte de façon troublante. Mais l'une, étant de lignage insigne, jouissait d'une très haute estime, et personne ne se serait autorisé à la traiter avec dédain : tous les égards, toutes les attentions lui étaient naturellement acquis. Tandis que l'autre, victime de la réprobation générale, dans quel malheur la faveur du souverain ne l'avait-elle pas entraînée! Certes, son chagrin restait entier, mais des sentiments nouveaux naissaient en lui, et qu'il y trouvât le plus grand des réconforts était dans l'ordre des choses.

Le prince Genji ne quittait guère son père, et celle que Sa Majesté honorait de fréquentes visites parvenait, moins que les autres, à se garder hors de sa vue. Toutes les épouses étaient d'une grande beauté - aucune, d'ailleurs, n'aurait accepté de se reconnaître inférieure aux autres -, mais elles étaient plus mûres que celle-ci, si jeune, si gracieuse! Il arrivait que le prince, même furtivement, l'aperçût, bien qu'elle s'efforçât à tout prix de demeurer invisible. Il ne gardait pas même l'ombre d'un souvenir de sa mère, mais à entendre la maîtresse en second du service du palais assurer que la jeune dame lui ressemblait de manière frappante, il avait dans son tendre cœur l'impression de la reconnaître et en venait à souhaiter être toujours auprès d'elle de façon à ce que, devenu un proche, il fût en mesure de la contempler. L'empereur portait un amour sans limites à l'un comme à l'autre. "Ne le fuyez pas. Chose étrange, je ne peux m'empêcher de voir en vous sa mère. Ne jugez donc pas que le prince vous manque d'égards, et chérissez-le. Il tient tant de sa mère par les traits du visage et par le regard que ce n'est pas sans raison si l'on décèle une telle ressemblance entre lui et vous ", disait-il à la dame pour plaider la cause du prince, si bien que celuici, avec son cœur d'enfant, prenait prétexte de la moindre occasion, fleur de printemps ou feuille d'automne, pour lui témoigner ses sentiments. L'affection toute particulière qu'il lui vouait ainsi déplaisait à la dame du Kokiden qui n'appréciait guère la nouvelle venue, et le ressentiment qu'elle éprouvait depuis toujours envers le prince s'en trouvant ranimé, elle conçut à son égard une acrimonie d'autant plus vive. À ses yeux, la beauté de son fils, le prince héritier, d'ailleurs célébrée par tous, était sans pareille, or elle n'était rien à côté de celle, rayonnante, du prince Genji : on appelait ce dernier Hikaru, le «prince de lumière ». De la même manière, comme la dame du clos de la Glycine était l'objet de la part du souverain d'un amour comparable à celui qu'il portait à l'enfant, on l'appela Kagayaku-hi no miya, la « princesse du jour étincelant ».

Bien que l'empereur répugnât à voir le prince quitter son habit d'enfant, il lui fit, lors de sa douzième année, revêtir la tenue virile ${ }^{31}$. Prenant lui-même en main l'organisation de la cérémonie, il en rehaussa la magnificence au-delà de ce que prévoyaient les règles. La cérémonie ne devait pas, suivant sa volonté, avoir moins de faste et d'éclat que celle qui s'était déroulée l'année précédente dans le pavillon du $\mathrm{Sud}^{32}$ en l'honneur du prince héritier. Il donna des ordres exprès afin que tout fût 
parfait jusque dans le moindre détail pour les différents banquets, car il craignait que le service normalement attendu de l'office des magasins de la cour et des greniers impériaux dans ces circonstances, ne révélât quelque négligence ${ }^{33}$. Un siège tourné vers l'Est, face auquel on disposa les nattes pour l'enfant et le ministre officiant fut installé pour le souverain dans la galerie orientale du Seiryōden. A l'heure du singe ${ }^{34}$, le prince Genji se présenta. Avec ses longs cheveux noués en boucle sur les tempes, l'éclat de sa grâce enfantine était tel qu'on regrettait qu'il dût y renoncer. Le chef du département du trésor procéda à la coupe des cheveux. Comme il lui coûtait de sacrifier une si belle chevelure! L'empereur se dit que si la mère de l'enfant avait vu ce spectacle..., mais il sut se rendre maitre de ses sentiments et, surmontant sa peine, il se ressaisit. Une fois la cérémonie de la coiffure terminée, le prince se retira pour revêtir sa nouvelle tenue, puis descendit dans le jardin faire les salutations rituelles à Sa Majesté: toute l'assistance avait les larmes aux yeux. Pour le souverain, la douleur se faisait plus vive que pour quiconque, car le souvenir des temps passés qu'il était parfois parvenu à oublier, revenait le submerger. Le prince était encore si jeune, le changement de coiffure n'allait-il pas lui faire perdre sa grâce? s'était-il souvent demandé. Or, désormais, une beauté bouleversante s'était ajoutée à son charme.

Le ministre de la gauche, chargé d'officier pour la coiffure, avait un souci : l'unique fille qu'il avait eue d'une de ses épouses, princesse impériale, et qu'il chérissait par-dessus tout, était convoitée par le prince héritier, alors qu'il avait conçu le dessein de la marier au prince Genji. Il s'en était ouvert à l'empereur pour solliciter son avis. "Eh bien, puisqu'il semble qu'aucune dame n'ait été désignée pour s'occuper du prince, qu'elle lui tienne donc compagnie la nuit de la cérémonie ». Le ministre s'était donc rangé à cette proposition de Sa Majesté.

Le prince se retira une nouvelle fois, et quand commencèrent les libations, il vint prendre place à l'extrémité de la rangée réservée aux fils de l'empereur. Le ministre en profita pour lui faire part à mots couverts des dispositions qui avaient été prises, plongeant dans l'embarras ce très jeune prince, qui ne répondit rien. C'est alors qu'une messagère ${ }^{35}$ envoyée par le souverain vint mander le ministre. Quand celui-ci se présenta, une dame au service de Sa Majesté lui remit selon l'usage des présents de circonstance : un ample vêtement blanc ainsi qu'une tenue de cour complète. Lors de l'échange des coupes, l'empereur s'ouvrit à lui de son inquiétude ${ }^{36}$ avec le poème suivant :

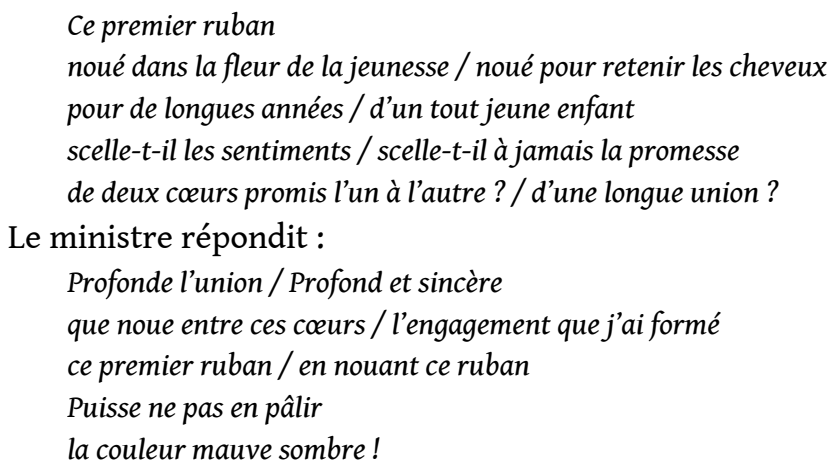

Puis il descendit par la longue galerie dans la cour et y exécuta les salutations rituelles. L'empereur lui fit tenir un cheval des écuries de la gauche, ainsi qu'un faucon provenant de la chancellerie privée. Les princes et les hauts dignitaires alignés au pied des escaliers reçurent des gratifications, chacun selon son rang. Le grand contrôleur de droite avait été chargé de préparer les plateaux de mets divers et les paniers de fruits 
qui devaient être offerts au souverain. On ne savait plus où mettre ni la nourriture ni les coffres contenant les récompenses destinées aux subalternes, et l'abondance surpassait même celle que l'on avait vue lors de la prise de coiffure virile du prince héritier. Excessif en la circonstance, le faste déployé était sans limites. Le soir venu, c'est à la résidence du ministre que, sur l'ordre de l'empereur, le seigneur Genji se retira. Il y fut accueilli avec une rare magnificence et entouré de mille prévenances. Sa grâce encore si enfantine éveillait chez chacun une profonde émotion. La demoiselle, plus âgée de quelques années, éprouvait, elle, de la gêne devant l'extrême jeunesse de son mari avec lequel elle se trouvait mal assortie.

Le ministre était tenu dans la plus grande estime par l'empereur, tandis que la princesse, mère de la demoiselle, était une sœur germaine du souverain, si bien que, d'un côté comme de l'autre, leur maison jouissait d'une position sans égale dans le monde. L'arrivée du jeune seigneur Genji ajoutait encore à cet éclat, et le prestige du ministre de la droite, aïeul du prince héritier et à ce titre destiné à exercer un jour le pouvoir, s'en trouvait considérablement amoindri. Le ministre de la gauche avait eu de nombreux enfants de ses différentes épouses, dont le capitaine de la garde attaché à la chancellerie privée que lui avait donné la princesse. Sa jeunesse et sa grande beauté avaient amené le ministre de la droite à arranger, malgré l'inimitié existant entre leurs familles, son mariage avec sa quatrième fille, chérie entre toutes. Comme le Genji, le jeune homme recevait le meilleur accueil de la part de son beau-père : on ne pouvait qu'envier les relations que tous deux entretenaient avec les familles de leurs épouses !

31 Le seigneur Genji, dont l'empereur exigeait constamment la présence au palais, n'avait guère le loisir de séjourner à la résidence du ministre. Au fond de son cœur, il ne cessait de penser à la dame du clos de la Glycine : sa grâce lui paraissait sans égale, c'est une épouse comme elle qu'il aurait voulue, aucune ne pouvait soutenir la comparaison avec elle. La demoiselle, fille du ministre, était, certes, d'une grande distinction et avait reçu l'éducation la plus soignée, mais il n'éprouvait aucune inclination envers elle, et un seul objet occupait, jusqu'à la souffrance, son jeune cœur. Depuis qu'il avait pris la coiffure virile, il ne lui était plus possible de l'approcher en pénétrant comme avant derrière les stores. Cependant, il pouvait s'entretenir avec elle par le truchement de la flûte et de la cithare à l'occasion des divertissements musicaux, et surprendre le son étouffé de sa voix était pour lui une consolation qui faisait du palais l'unique séjour auquel il aspirait. Il s'y attardait jusqu'à cinq ou six jours à la suite, ne revenant que par intermittence, pour deux ou trois jours, à la résidence du ministre où l'on excusait son comportement, le mettant sur le compte de son jeune âge, et où l'on continuait à l'entourer d'attentions. Le ministre avait engagé pour le service du prince et de son épouse les dames les plus élégantes, choisies avec le plus grand soin. Il multipliait les divertissements musicaux propres à attacher son cœur et ne ménageait pas sa peine pour lui complaire en toute chose. Au palais, le Genji continuait à disposer des appartements du clos du Paulownia, où l'on avait maintenu sans les laisser se disperser les dames qui avaient servi autrefois sa mère. Quant à la résidence familiale de celle-ci, ordre fut donné par l'empereur au service des réparations de même qu'à l'office des fabrications de la restaurer magnifiquement. L'étang fut agrandi pour rehausser la beauté des bosquets et des collines artificielles, et l'endroit s'anima du bruit des travaux. «Ah! si seulement je pouvais y vivre avec une épouse selon mon cœur!» ne cessait de soupirer le prince. Le surnom de seigneur Hikaru lui avait été donné en forme d'éloge par les envoyés venus de Koma, à ce que l'on rapporte. 


\section{NOTES}

1. Yang Guifei (jap. Yō Kihi 楊貴妃, 719-756), la « concubine impériale Yang » (de son vrai nom Yang Taizhen 楊玉環). Sous la dynastie des Tang (618-907), l'empereur Xuanzong (r. 712-756) s'éprit d'elle jusqu'à, d'après la tradition, en oublier les affaires publiques. C'est pourquoi, lors de la « révolte d'Anshi » (755), les soldats de la garde de l'empereur exigèrent sa mise à mort.

2. Le Kiritsubo ou "clos du Paulownia ", également appelé Shigeisha ou «clos du Paysage frais comme le printemps" est situé dans l'enceinte de la résidence impériale, au nord-est. (cf. Francine Hérail, Notes journalières de Fujiwara no Michinaga ministre à la cour de Heian, tome 1, Librairie Droz, 1987, p. 121 et 123). Il est d'usage de désigner les femmes vivant à la cour par le nom du pavillon qu'elles occupent. Pour tout ce qui concerne l'emplacement et la description des lieux cités, voir l'article de Marie Maurin «Sur les pas du Genji : le palais de Heian », p. 185 à 226 de ce numéro.

3. Le Kōrōden (ou Kōryōden) se trouve à l'arrière du Seiryōden, le "pavillon de Pureté et de Fraîcheur » où se passait la vie quotidienne de l'empereur (cf. Francine Hérail, op.cit., p. 121-122).

4. Le palanquin dont il est question, voiture fermée à deux roues tirée à bras d'hommes, n'était autorisé à circuler dans l'enceinte de la résidence impériale que sur ordre exprès de l'empereur. Ce mode de transport était en principe réservé aux personnages importants : princes, ministres, épouses impériales, etc. Il s'agit donc dans le cas présent d'une faveur exceptionnelle.

5. Kokiden est le "pavillon de l'Expansion du Beau» où habite cette épouse impériale. Le bâtiment est situé au nord du Seiryōden (cf. note 2).

6. Le nom exact de la dame est Yugei no myōbu, ce qui signifie «dame fonctionnaire du cinquième rang [dont le père, ou le mari est] garde des portes ». Il était fréquent de désigner une femme en service au palais par la fonction qu'exerçait un de ses proches de sexe masculin (père, frère, mari ou fils).

7. Expression empruntée à un poème d'amour anonyme recueilli dans le Kokin wakashū $\left(n^{\circ} 647\right)$ qui dit la déception ressentie après la concrétisation d'une relation amoureuse jusqu'alors fantasmée : « Notre rencontre fugitive / au sein de la nuit / d'un noir de myrtille / en rien n'a surpassé / le rêve que j'en avais fait ».

8. "Entrée principale » rend ici le "côté sud» de l'original. En effet, dans les résidences de l'aristocratie de ce temps, c'est au sud - face au jardin - que se trouvaient l'escalier d'honneur et les pièces de réception.

9. Le pin de Takasago est un symbole d'extrême longévité. Il s'agit là d'une allusion au poème recueilli dans le Kokin waka rokujō $\left(\mathrm{n}^{\circ} 3057\right)$ : «Je n'ai guère envie / de m'entendre dire: / comment, toujours en vie? / ce que pourrait penser le pin de Takasago/ me remplit de confusion "; le poète ne souhaite pas que l'exposition de sa longévité donne lieu à des remarques désobligeantes.

10. L'histoire tragique de Yang Guifei (cf. note 1) inspira le poète Bai Juyi 白居易 (jap. Haku Kyoi), (772-846), connu également sous le nom Bai Letian 白楽天 (jap. Haku Rakuten), qui sur ce sujet écrivit l'un des poèmes lyriques les plus célèbres de la littérature chinoise, le Changhen ge 長恨歌 (jap. Chōgon-ka), le "Chant des regrets éternels » (806). Ce long poème connut dès sa première diffusion une exceptionnelle popularité, et exerça une profonde influence sur la littérature chinoise postérieure, ainsi que sur la littérature japonaise de l'époque de Heian. Le traitement du thème de l'amour malheureux entre un empereur et l'une de ses concubines dans le Genji monogatari, qui s'appuie sur de nombreuses reprises littérales ou allusives du texte de Bai Juyi, constitue un exemple majeur de la réception de ce poème dans la littérature japonaise de l'époque. 
11. «Teiji no in» 亨子院 est l'un des noms posthumes (du nom de l'une de ses résidences) de l'empereur Uda 宇多 (867-931, r. 887 à 897). Imprégné de bouddhisme et passionné de poésie, il fut à l'origine de nombreuses entreprises littéraires d'envergure.

12. Selon les interprétations, soit l'empereur ne faisait que commander les peintures et les poèmes, soit il supervisait également la conception des tableaux.

13. Allusion au Chant des regrets éternels. Un an après la mort de Yang Guifei l'empereur, ne se résignant toujours pas à sa perte, envoie un magicien taoïste à sa recherche. Celui-ci la retrouve sur une montagne où séjourne un grand nombre d' "Immortelles ». Yang Guifei, qui avait reçu de l'empereur un coffret en émail et une épingle d'or comme gages d'amour, arrache le couvercle du coffret et brise l'épingle en deux ; elle donne ensuite ces deux fragments au messager pour qu'il les transmette à l'empereur, disant : «Si son cœur est aussi résistant que cet or et que cet émail / sur terre ou au ciel, nous nous retrouverons un jour. " (Bai Juyi, Chant des regrets éternels et autres poèmes, trad. Georgette Jaeger, Orphée / La Différence, 1992, p.37). On se souviendra qu’à l'occasion de la visite de Yugei, la mère de Kiritsubo avait envoyé à l'empereur quelques souvenirs de la disparue, dont un «nécessaire de coiffure ", d'où cette réflexion du souverain, qui associe les objets ayant appartenu à la défunte à l'écho de ce passage du poème chinois.

14. Le roman suit fidèlement le texte chinois : « ... l'empereur retrouve les étangs et les parcs, rien n'a changé / lotus sur l'étang Taiyi, saules dans le parc Weiyang / lotus pareils à son visage, feuilles de saule pareilles à ses sourcils / en proie aux souvenirs, il ne peut retenir ses larmes... » (trad.cit., p. 31).

15. Allusion au poème de Fujiwara no Masatada (Gosen wakashū, printemps, $n^{\circ} 212$ ), envoyé à un ami : «La splendeur des fleurs / et le chant des oiseaux / ne me touchent plus / [sans nouvelles de vous] c'est dans la peine / que vainement s'écoulent mes jours ».

Le Kawachi-bon donne la version suivante : «Elle avait dû être splendide dans sa parure à la mode chinoise avec son visage semblable "aux lotus de l'étang de Taiyi", mais quand l'empereur se rappelait le charme empreint de douceur et de fragilité de la défunte, plus délicate que les valérianes ployant au vent, plus aimable et attachante que les cillets luisants de rosée, il ne trouvait "ni fleurs ni oiseaux auxquels il pût la comparer"».

16. «... nous avons fait vœu d'être au ciel deux oiseaux qui n'ont qu'une paire d'ailes / et sur terre, deux branches entrelacées d'un même arbre » (trad. cit., p. 37).

17. Allusion au dernier vers du Changhen ge : «... le Ciel et la Terre dureront longtemps, mais un jour ils prendront fin / tandis que ce chagrin durera toujours - éternellement » (trad. cit., p. 37).

18. Le Kawachi-bon donne la version suivante : « la dame du Kokiden, qui, contrariée, ne se rendait plus dans ses appartements du Seiryōden ».

19. L'auteur emploie volontairement dans cette pièce des expressions qui imposent de lire certains vers de deux façons différentes.

20. L'allusion au Changhen ge est répartie sur deux phrases: «la mèche de l'unique lampe se consume sans qu'il trouve le sommeil » (trad. cit., p. 31).

21. Entre $1 \mathrm{~h}$ et $3 \mathrm{~h}$ du matin.

22. Allusion à une pièce que la poétesse Ise a composée sur le thème du Changhen ge : «Derrière les stores / sertis de perles nous dormions / ignorant l'aube / Pouvais-je alors deviner / que même en rêve je ne la verrai plus » (Ise shū $\left.n^{\circ} 55\right)$.

23. Cérémonie organisée quand un enfant de famille noble atteint l'âge de sept ou huit ans, et qui marque le début de son étude des textes chinois. Ordinairement, l'enfant reprenait la lecture d'un texte à haute voix faite par un conférencier. Le prince est donc encore très jeune, ce qui lui permet de s'introduire librement auprès des femmes de haut rang qui ne devaient pas s'exposer au regard masculin. Elles vivaient cachées derrière les stores, les paravents et les rideaux.

24. C'est-à-dire la cour impériale.

25. Koma est le nom d'un royaume situé au nord de la péninsule coréenne. 
26. L'auteur fait référence au texte rédigé par l'empereur Uda, constitué d'une série de recommandations destinées à son jeune fils Daigo (r. 897-930) sur le point d'accéder au trône.

27. Les Kōrokan « Hôtels des hôtes étrangers » servaient à l'origine pour l'accueil, le logement et l'approvisionnement des délégations étrangères en visite au Japon; à partir du $\mathrm{IX}^{\mathrm{e}}$ siècle, on $\mathrm{y}$ logea également des commerçants étrangers et on y faisait des affaires. Du Xe au XIII ${ }^{\mathrm{e}}$ siècle l'époque qui nous concerne - ces établissements servaient uniquement d'hôtels pour les commerçants. Deux hôtels de ce type ont été fondés dans la capitale Heian-kyō sous le règne de l'empereur Kanmu (r. 781-806) ; ils se trouvaient des deux côtés de l'avenue Suzaku, près des temples Tō-ji et Sai-ji (cf. Charlotte von Verschuer, Les Relations officielles du Japon avec la Chine aux $\mathrm{VIII}^{e}$ et IX ${ }^{e}$ siècles, Librairie Droz, 1985, p. 438-439).

28. "Selon le code, ont droit au titre de prince et à l'inscription dans un registre spécial cinq générations en comptant l'empereur souche de la lignée. Ceux qui venaient ensuite rejoignaient les rangs du peuple et recevaient un nom et éventuellement un titre. À partir de l'époque de Heian, les empereurs prirent l'habitude de donner à la plupart de leurs fils et de leurs petits-fils un nom, les plus courants sont Taira et Minamoto, et de les mettre ainsi à la tête de nouvelles lignées de fonctionnaires. Le sort de ces nouvelles lignées, bénéficiant au moins pour une ou deux générations de la protection toute spéciale de l'empereur, était plus enviable que celui de princes de la sixième ou septième génération rejetés dans le peuple sans protection. » (Francine Hérail, Notes journalières de Fujiwara no Sukefusa, tome 1, Librairie Droz, 2001, p. 133). «Genji » signifie littéralement «famille Gen » (lecture sino-japonaise de " Minamoto »), et marque donc l'origine impériale de la lignée. Ajoutons enfin qu'il fallait un ordre impérial pour qu'un enfant d'empereur soit déclaré prince impérial, et qu'il devait attendre la célébration de la majorité pour recevoir un rang impérial qui lui assure un revenu régulier.

29. Les connaissances requises pour devenir un homme politique compétent sont le droit, les procédures administratives, le protocole, les précédents, les lettres...

30. 30 Le Kawachi-bon donne la version suivante: «L'empereur réitéra alors son invitation d'une manière encore plus pressante. "Pourquoi restez-vous dans cet état précaire? Laissez-moi vous considérer à l'égal des princesses mes filles." "

31. Cérémonie au cours de laquelle le prince héritier va être déclaré majeur, ce qui se marque par des modifications dans son costume et dans sa coiffure. Il va quitter la coiffure des jeunes garçons - les cheveux en deux touffes de chaque côté de la tête - pour prendre celle des hommes adultes, les cheveux relevés en une sorte de chignon sur lequel se pose le bonnet de cour (cf. Francine Hérail, op.cit., tome 2, 2004, p. 469). Cette cérémonie concernait tous les jeunes garçons de l'aristocratie. Dans le cas de la famille impériale, on la célébrait généralement entre 11 et 16 ans.

32. Le pavillon du Sud, Nanden, également appelé Shishinden " pavillon de l'étoile Polaire », était le bâtiment où se déroulait une bonne part de la vie publique du souverain (cf. Francine Hérail, Notes journalières de Fujiwara no Michinaga, tome 1, Librairie Droz, 1987, p. 122).

33. Le Kawachi-bon donne la version suivante : « Même pour le service normalement simplifié de l'office des magasins de la cour et des greniers impériaux dans ces circonstances, il donna des ordres exprès... ».

34. Entre 3 h et 5 h de l'après-midi.

35. Il s'agit d'une naishi no jō, ou «fonctionnaire de troisième classe du service intérieur du palais » (cf. Francine Hérail, La Cour et l'administration du Japon à l'époque de Heian, Paris, École Pratique des Hautes Études ; Genève, Droz, 2006, p. 503 et passim).

36. L'empereur exprime par son poème des vœux pour la pérennité du mariage entre le prince et la fille du ministre, qui va lui tenir compagnie cette nuit. Habituellement, la jeune fille destinée à devenir l'épouse légitime accomplit ce rôle, et selon la coutume, le futur époux doit se rendre chez elle trois nuits de suite pour que le mariage soit déclaré officiel. 


\section{RÉSUMÉS}

Traduction du $1^{\mathrm{er}}$ chapitre du Genji monogatari (Kiritsubo), travail collectif des chercheurs du CEJ (Inalco) et du Greja (Paris 7), basée sur l'édition Shinchō Nihon Kotei Shūsei.

Translation of the 1st chapter of Genji monogatari (Kiritsubo), collective work of CEJ (Inalco) and Greja (Paris Diderot University) researchers, based on the Shinchō Nihon Kotei Shūsei edition.

INDEX

Thèmes : littérature, traductologie

キーワード : Genji monogatari 源氏物語, Murasaki Shikibu 紫式部 (v. 973-v. 1014 ou 1025), Heian jidai 平安時代 (794-1185), bungaku 文学, hon.yaku kenkyū 翻訳研究

Mots-clés : Dit du Genji, Murasaki Shikibu (v. 973-v. 1014 ou 1025)

Keywords : Genji monogatari, Heian, Murasaki Shikibu (v. 973-v. 1014 or 1025), Literature, Translation Studies

Index chronologique : Heian 